\title{
Recomendaciones sobre el manejo en psoriasis y COVID-19: opinión de expertos
}

\author{
Juan Raúl Castro-Ayarza'; Jhyld Carolaind Camacho-Barbosa²; Manuel Darío Franco- \\ Franco3; Carolina Cárdenas-Henao $^{4}$; Julio Roberto Amador'5; Susana Chiquito-García6; \\ Jorge Luis Bermúdez-Flórez ${ }^{7}$; Catalina Orozco-González ${ }^{8}$; Jorge Hernando Donado- \\ Gómez $^{9}$; Natalia Duque-Zapata ${ }^{10}$
}

\section{RESUMEN}

La enfermedad por coronavirus 2019 (COVID-19) puede impactar en la estrategia terapéutica de la psoriasis, dado que estos pacientes, por su daño extenso en piel y los posibles efectos inmunosupresores o inmunomoduladores de las terapias biológicas, podrían estar en un mayor riesgo de infección por el virus. Por tanto, el objetivo de esta opinión de expertos basada en la revisión narrativa de la literatura es establecer recomendaciones generales sobre la atención ambulatoria y el tratamiento de pacientes con psoriasis durante la pandemia por COVID-19. Se evidenció que en los pacientes con psoriasis y COVID-19 se debe evaluar cuidadosamente, caso por caso, la relación riesgo-beneficio de cualquier intervención terapéutica inmunosupresora. En conclusión, se requiere de estudios con mayor nivel de evidencia para comprender el papel de la respuesta inmunitaria en el resultado de la COVID-19 en estos pacientes.

PALABRAS CLAVE: dermatología; COVID-19; coronavirus; psoriasis; terapia con medicamentos.

1. Dermatólogo, Grupo de Dermatología, Medicarte, Colombia. ORCID https://orcid.org/oooo-0oo2-0113-2684

2. Magíster en Epidemiología, Grupo de Investigación Medicarte (GIM), Medicarte, Colombia. ORCID https://orcid.org/oooo-0003-4405-3713

3. Dermatólogo, Grupo de Dermatología, Medicarte, Colombia. ORCID https://orcid.org/oooo-0002-2476-4991

4. Dermatólogo, Grupo de Dermatología, Medicarte, Colombia. ORCID https://orcid.org/oooo-0001-5437-0187

5. Dermatólogo, Grupo de Dermatología, Medicarte, Colombia. ORCID https://orcid.org/oooo-0002-2476-4991

6. Dermatólogo, Grupo de Dermatología, Medicarte, Colombia. ORCID https://orcid.org/ooo-ooo1-6281-2918

7. Dermatólogo, Grupo de Dermatología, Medicarte, Colombia. ORCID https://orcid.org/oooo-0oo1-6972-921X

8. Magíster en Epidemiología, Grupo de Investigación Medicarte (GIM), Medicarte, Colombia. ORCID https://orcid.org/ oooo-0001-7845-7279

9. Magíster en Epidemiología, Grupo de Investigación Medicarte (GIM), Medicarte, Colombia. ORCID https://orcid.org/oooo-0001-8581-2267

10. Magíster en Epidemiología, Grupo de Investigación Medicarte (GIM), Medicarte, Colombia. ORCID https://orcid.org/oooo-0002-3242-8920

Correspondencia: Jhyld Carolaind Camacho-Barbosa; email: jcamacho@medicarte.com.co

Recibido: $12 / 06 / 20$; aceptado: $27 / 07 / 20$

Cómo citar: Castro-Ayarza JR, Camacho-Barbosa JC, Franco- Franco, Cárdenas-Henao C, Amador JR, Chiquito-García S, BermúdezFlórez JL, Orozco-González C, Donado-Gómez JH, Duque-Zapata N. Recomendaciones sobre el manejo en psoriasis y COVID-19: opinión de expertos. Rev Asoc Colomb Dermatol. 2020;28(2): 138-147. DOI:

Financiación: ninguna, conflictos de interés: El grupo trabaja en medicarte es una institución que se encarga de la atención, formulación y despacho de terapias biológicas. Juan Raúl Castro-Ayarza ha recibido honorarios o patrocinios de Abbvie, Biopas, Janssen, Novartis y Sanofi. Manuel Darío Franco-Franco ha recibido honorarios o patrocinios de Abbvie, Eli-Lilly, Janssen y Novartis. Carolina CárdenasHenao ha recibido patrocinios de Janssen. Julio Roberto Amador ha recibido honorarios o patrocinios de Eli-Lilly,GSK, Janssen, Novartis y Sanofi. Susana Chiquito-García ha recibido honorarios o patrocinios de Janssen y Novartis. Jorge Luis Bermúdez-Flórez ha recibido honorarios o patrocinios de Abbvie y Pfizer. 


\section{MANAGEMENT RECOMMENDATIONS OF PSORIASIS AND COVID-19: EXPERT OPINION}

\section{SUMMARY}

Coronavirus disease 2019 (COVID-19) may impact the therapeutic strategy of psoriasis, due to the extensive skin damage and the possible immunosuppressive or immunomodulatory effects of biological therapies, could be an increased risk of virus infection, on these patients; therefore, the aim of this expert opinion based on a narrative literature review is to present general recommendations on ambulatory care and treatment of psoriasis patients, during the COVID-19 pandemic. It is evident that psoriasis patients with COVID-19, should be evaluated for the risk-benefit ratio of any immunosuppressive therapeutic intervention. In conclusion, studies with a higher level of evidence are required to understand the role of the immune response in COVID-19 result in these patients.

KEY WORDS: Dermatology; COVID-19; Coronavirus; Psoriasis; Drug therapy.

\section{INTRODUCCIÓN}

La enfermedad por coronavirus 2019 (COVID-19) que causa el síndrome respiratorio agudo grave (SARSCoV-2) puede tener un impacto en la estrategia terapéutica de un trastorno complejo como la psoriasis, cuyo compromiso extenso en piel afecta la salud del paciente por la respuesta inflamatoria sistémica que acompaña la enfermedad, lo que podría generar un mayor riesgo de infección en comparación con la población general. Existe una preocupación por los efectos inmunosupresores o inmunomoduladores que podrían hacer que los pacientes que reciben terapias biológicas sean más susceptibles a la infección por COVID-19. Este es el caso del tratamiento de la psoriasis y de otras enfermedades dermatológicas, y aunque la mayoría de los expertos están de acuerdo en que la relación riesgo-beneficio está a favor de mantener terapias biológicas selectivas debido al riesgo de complicaciones derivadas de la enfermedad sin tratamiento (por ejemplo, eritrodermia), es importante tener en cuenta la susceptibilidad del paciente a la infección por COVID-19, que pese a que todavía no hay datos específicos sobre esta, existen datos sobre complicaciones infecciosas respiratorias y sistémicas para los pacientes en tratamiento con terapias biológicas en los ensayos pivotales para la psoriasis ${ }^{(1-4)}$.

Por otra parte, se ha reportado que en los pacientes con SARS-CoV-2 han aumentado las concentraciones plasmáticas de citocinas relacionadas con la inflamación, las cuales están involucradas en la etiopatogenia de la psoriasis, lo que sugiere que la COVID-19 puede ser una nueva entidad que exacerba la psoriasis $\operatorname{vulgar}^{(5,6)}$.

\section{OBJETIVO}

Establecer recomendaciones generales, clínicas y administrativas sobre la atención ambulatoria y el tratamiento de pacientes con psoriasis durante la pandemia por COVID-19, según la evidencia disponible y la opinión de expertos.

\section{METODOLOGÍA}

Se realizó una revisión de la literatura científica en la base de datos PubMed, y literatura gris en el navegador de Google, además de lo publicado por las asociaciones en dermatología y psoriasis colombiana, española y americana, con límites de búsqueda de artículos publicados en el último año, en idioma inglés y español. La estrategia de búsqueda incluyó vocabulario controlado explotado (MeSH), ("psoriasis"[MeSH Terms] OR "psoriasis"[All Fields]) AND (((((("COVID-19"[All Fields] OR "COVID-2019"[All Fields]) OR "severe acute respiratory syndrome coronavirus 2"[Supplementary Concept]) OR "severe acute respiratory syndrome coronavirus 2"[All Fields]) OR "2019-nCoV"[All Fields]) OR "SARS-CoV-2"[All Fields]) OR "2019nCoV"[All Fields]) AND ("coronavirus"[MeSH Terms] OR "coronavirus"[All Fields])); y lenguaje libre, considerando sinónimos, abreviaturas, variaciones ortográficas y plurales. 
Se extrajo la evidencia más relevante y se elaboró un informe científico con las recomendaciones y componentes identificados en la revisión, los cuales fueron sometidos a evaluación por el equipo de trabajo de expertos metodológicos (3 epidemiólogos) y temáticos (6 dermatólogos), con calificación tipo Likert para cada recomendación (tabla 1); definiendo como fundamentales las incluidas aquí (véase anexo 1). Esta es la opinión de un grupo de expertos basado en revisión narrativa de literatura.

Por tratarse de un estudio basado en fuentes secundarias, sin acceso a información confidencial, se considera un estudio sin riesgo de acuerdo con la resolución 8340 del Ministerio de Salud.

La selección de expertos metodológicos y temáticos que han declarado de forma transparente sus conflictos de interés y la transparencia en los análisis metodológicos permiten afirmar que es un estudio ceñido a la ética de la investigación.

\section{Población objeto}

Pacientes con diagnóstico de psoriasis:

1. Con objetivo terapéutico logrado.

2. Sin objetivo terapéutico logrado.

3. Con buen pronóstico global.

4. Con mal pronóstico global: alto riesgo o con compromiso sistémico (articular, gastrointestinal, entre otros), > 60 años, presencia de comorbilidades, polimedicación, compromiso en la calidad de vida (PASI y DLQI elevados), bajos recursos económicos y bajo nivel de educación.

\section{PREVENCIÓN DE LA INFECCIÓN POR COVID-19 EN PACIENTES DERMATOLÓGICOS}

Debido al alto riesgo de transmisión nosocomial de la COVID-19, investigadores de China realizaron un planteamiento del manejo de emergencias para prevenir y controlar la infección nosocomial del virus en los departamentos de dermatología, donde se plantea que los pacientes tienen lesiones en la piel que podrían facilitar la transmisión del virus por contacto indirecto, situación incierta que se puede abordar mediante prevención. Las estrategias planteadas incluyeron ${ }^{(7)}$ :

- $\quad$ Establecer estaciones de preexamen y triaje en la entrada del hospital y el área de consulta, en las cuales las enfermeras y un dermatólogo (según necesidad) evalúan los síntomas de COVID-19. Cuando hay fiebre, se debe activar el protocolo COVID-19 y no se puede realizar consulta presencial; después de asegurar que la fiebre es causada por una enfermedad de la piel, el dermatólogo participa en la consulta y los pacientes que se determine que no están infectados por el virus pueden acceder al servicio de dermatología.

- Uso del tapabocas y el lavado correcto de manos, de paciente y médico, durante la consulta.

- $\quad$ Consulta en línea o virtual para pacientes leves y sin emergencia, y seguimiento por correo electrónico por solicitud de imágenes de las lesiones cutáneas ${ }^{(8,9)}$.

- Conformación de un grupo de contingencia para prevenir y controlar el brote de COVID-19, quienes discuten e informan diariamente sobre las condi-

Tabla 1. Nivel de consenso según mediana de calificación Likert de las recomendaciones

$\begin{array}{ll}\text { Mediana de calificación } & \begin{array}{l}\text { Consenso de la recomendación } \\ \text { evaluada }\end{array} \\ 1 \text { a } 3 & \text { En contra } \\ 4 \text { a } 6 & \text { No hay consenso } \\ 7 \text { a9 } & \text { Afavor }\end{array}$


ciones actualizadas de los pacientes sospechosos de la infección.

- Aislamiento, notificación y transferencia de pacientes sospechosos según las políticas y procesos locales de control de infecciones.

\section{TRATAMIENTO DE LOS PACI- ENTES DERMATOLÓGICOS DU- RANTE LA PANDEMIA}

Entre los aspectos importantes que podrían guiar la decisión de continuar o no con la terapia biológica durante la pandemia, se incluye tener en cuenta que en pacientes con COVID-19 grave se produce una liberación de grandes cantidades de citocinas proinflamatorias, incluidas algunas de las sobreexpresadas en psoriasis, como el factor de necrosis tumoral alfa (FNT- $\alpha$ ) y la interleucina 33 (IL-33), en particular durante el síndrome de dificultad respiratoria aguda, que es la principal causa de muerte por la infección ${ }^{(2)}$.

De acuerdo con las revisiones de literatura, se aconseja que durante la pandemia por COVID-19 los dermatólogos prioricen e individualicen los protocolos de tratamiento para pacientes con psoriasis según la gravedad de la enfermedad, las condiciones clínicas y la gravedad del virus. Para aquellos con mayor riesgo, con comorbilidades cardiovasculares y pulmonares, el riesgo-beneficio puede favorecer la interrupción según el caso ${ }^{(2,10)}$. Además, los biológicos tienen una vida media prolongada, por lo que no es razonable suspenderlos durante unas pocas semanas, dado que la pandemia es probable que dure un tiempo prolongado y el paciente podría presentar una recaída con riesgo de su salud u hospitalizaciones no necesarias ${ }^{(11)}$. Sin embargo, también es importante tener en cuenta que las tasas de infección respiratoria en pacientes con medicamentos biológicos antes de la pandemia fueron comparables a las del placebo y, por el contrario, la interrupción de algunos biológicos puede provocar la pérdida de respuesta cuando se reintroducen los tratamientos o incluso dar lugar a la formación de anticuerpos contra el biológico descontinuado $(4,6,10)$.

En un documento desarrollado por Price y colaboradores, en el cual tenían como objetivo proporcionar información reciente sobre la COVID-19 y cómo realizar el abordaje terapéutico de los pacientes dermatológicos con el uso de inmunomoduladores/inmunosupresores, recomendaron que si el paciente está con inmunosupresores clásicos (corticoesteroides, ciclosporina, entre otros), antimetabolitos (azatioprina, metotrexato, entre otros) o rituximab, se debe detener la terapia cuando se presenten síntomas virales, especialmente con exposición conocida o potencial. En los pacientes tratados con infliximab, etanercept, certolizumab, adalimumab, anakinra, brodalumab, secukinumab, ixekizumab, ustekinumab y guselkumab recomiendan continuar con la terapia si los síntomas virales son leves; sin embargo, se debe considerar detener el tratamiento si los síntomas virales empeoran o se desarrolla fiebre alta; y finalmente para los pacientes en tratamiento con dupilumab y apremilast, recomiendan continuar a menos que presenten síntomas graves. En acuerdo con los pacientes es posible considerar la descontinuación del tratamiento en aquellos con enfermedad estable sin inmunosupresores $^{(12)}$.

En un reporte de caso de un paciente con obesidad, quimioterapia reciente, persistencia de oligodendroglioma cerebral y contagio viral por COVID-19, que estaba en tratamiento con apremilast (medicamento no disponible en Colombia), se observó que el paciente se recuperó en 6 días, aunque no se suspendió la terapia y fue dado de alta hospitalaria después de dos pruebas negativas para COVID-19. Además, se evidenció que el medicamento ofreció un control aceptable de la psoriasis, limitado a escamas leves y eritema, especialmente en el tronco, y concluyeron que el apremilast es un medicamento seguro, que no interfiere con la infección por COVID-19 ${ }^{(1)}$.

En una revisión de la literatura hecha por Amerio y colaboradores, en la cual analizaron la evidencia sobre los mecanismos patogénicos similares de enfermedad grave y factores de riesgo para SARS-CoV, síndrome respiratorio de Oriente Medio (MERS) y SARS-CoV-2, y como la condición inmunosupresora podría predisponer a enfermedades más graves en pacientes infectados por SARS-CoV-2, los autores concluyeron que el tratamiento de pacientes psoriásicos con biológicos no debe suspenderse durante el tiempo de la pandemia y que la precaución debe centrarse en pacientes de edad avanzada con morbilidades coexistentes, como hipertensión, diabetes y obesidad, las cuales se ha demostrado que, en caso de infección por COVID-19, aumentan las probabilidades de desarrollar una enfermedad grave. También recomiendan que en este grupo de pacientes la suspensión del tratamiento solo se debe considerar cuando estos desarrollan síntomas similares a los de la gripe o específicos de la COVID-19 
y si están expuestos a un contacto de alto riesgo con personas infectadas por el virus ${ }^{(6)}$.

De acuerdo con un estudio realizado en Italia por Conforti y colaboradores en pacientes con psoriasis, el tratamiento con ciclosporina, metotrexato y anti-FNT-a debería ser cuidadosamente evaluado, dado que estos medicamentos podrían causar una disminución de la respuesta inmunitaria y mayor susceptibilidad a infecciones potencialmente mortales como la COVID-19. Por tanto, recomiendan limitar y/o reducir el tiempo de administración y preferir los medicamentos tópicos y/o con menor impacto en el sistema inmunitario; además, sugieren suspender toda terapia inmunosupresora y biológica donde exista exposición confirmada a la COVID-19 ${ }^{(13)}$. Por su parte, otros investigadores italianos, como Bardazzi y colaboradores y Coletto y colaboradores, encontraron que estas recomendaciones pueden ser controversiales, dada la falta de evidencia científica y que según las indicaciones oficiales, si el paciente está estable o saludable, no está indicado suspender la terapia inmunosupresora/ inmunomoduladora, ya que el riesgo de reactivación de la patología subyacente podría agregar un factor de riesgo adicional a las infecciones, como la COVID-19. Concluyen que la interrupción de la terapia biológica en un paciente con psoriasis podría implicar un desequilibrio de las citocinas inflamatorias que no solo exacerba la psoriasis, sino que también está involucrada en la patogénesis de infecciones virales ${ }^{(14,15)}$.

En un reporte realizado por Carugno y colaboradores acerca de la experiencia sobre el manejo de pacientes con psoriasis tratados con terapia biológica en Bérgamo, Italia, un área de alta incidencia para COVID19, en donde identificaron 25 casos sospechosos para el virus y, como precaución, estudiando caso por caso, suspendieron la terapia en 6 de ellos por reporte de síntomas respiratorios hasta 30 días después de que los síntomas se resolvieron, observaron que no se reportaron casos graves de infección por COVID-19 e incluso los pacientes que continuaron la terapia mostraron síntomas principalmente leves ${ }^{(16)}$.

En una serie de casos de pacientes con psoriasis mayores de 60 años $(n=4)$, con comorbilidades como hipertensión y diabetes, entre otras, tratados con biológicos y que tuvieron contacto de riesgo con COVID19, observaron que 2 de estos no presentaron síntomas de la enfermedad y no requirieron cambios en la terapia; sin embargo, los otros 2 casos tuvieron confirmación diagnóstica para el virus y se les interrumpió la terapia biológica durante el período de aislamiento, sin observarse empeoramiento de la psoriasis; ambos pacientes permanecieron en remisión completa, por lo cual concluyeron que es posible que el tratamiento continuo con medicamentos biológicos podría desempeñar un papel protector contra el inicio y la evolución de la infección; sin embargo, se necesitan más estudios para investigar la hipótesis ${ }^{(17)}$.

En un artículo de Torres y colaboradores, en el cual se discute el manejo de enfermedades cutáneas inmunomediadas durante la pandemia de COVID-19, los autores concluyen que los datos disponibles sobre brotes pasados y presentes de coronavirus sugieren que los pacientes inmunodeprimidos no tienen un mayor riesgo de enfermedad grave ni complicaciones en comparación con la población general, y que los medicamentos inmunosupresores e inmunomoduladores podrían controlar potencialmente la "tormenta de citocinas" asociada a un peor resultado en estos pacientes. Por tanto, sugieren que pueden continuar su tratamiento y evitar así aumentar el riesgo, la discapacidad, la mala calidad de vida y el uso de servicios de salud. Sin embargo, también indican suspender la inmunosupresión del tratamiento biológico en pacientes con infección activa por COVID-19, y en aquellos que desarrollan síntomas relacionados con el virus, o son contactos cercanos de casos confirmados, se debe considerar individualmente el aumento de los intervalos de administración o la interrupción temporal del tratamiento ${ }^{(18)}$.

De acuerdo con los resultados de ensayos clínicos de fase III sobre terapia biológica en psoriasis y tasas de infección respiratoria superior, influenza e infección grave, investigadores norteamericanos establecieron recomendaciones de asesoramiento al paciente dermatológico, entre las cuales están: lavado de manos, distanciamiento social, evaluación individualizada sobre el inicio o continuación de la terapia biológica según factores de riesgo. En los casos que se requieran modificaciones, considerar las diferentes opciones de manejo como interrupción temporal, reducción de la frecuencia de la dosis, transición a una alternativa biológica, reducción o interrupción de inmunosupresores concomitantes (metotrexato) y aumento en el uso de agentes tópicos, fototerapia $\mathrm{u}$ otros medicamentos no inmunosupresores; $y$, finalmente, en los pacientes que resulten positivos para COVID-19 mantener la dosis hasta que la infección desaparezca ${ }^{(19)}$. 
En dos reportes de casos de pacientes con psoriasis tratados con inhibidores IL-23 e IL-17 con diagnóstico positivo para COVID-19 asintomáticos, los autores sugieren que la inhibición del eje IL-23/IL-17 podría no ser perjudicial en el contexto de la infección por el virus; sin embargo, se requiere mayor evidencia para respaldar la hipótesis ${ }^{(20,21)}$.

En un estudio observacional multicéntrico retrospectivo que incluyó pacientes con psoriasis en placas ( $\mathrm{n}=$ 5206) tratados con terapia biológica y en el cual tenían como objetivo reportar el número de pacientes hospitalizados o muertos por infección con COVID-19, observaron que no se presentaron muertes por el virus y solo 4 pacientes fueron hospitalizados por neumonía intersticial relacionada con este, por lo cual concluyeron que aunque los pacientes con psoriasis generalmente presentan comorbilidades metabólicas y cardiovasculares y, además, son tratados con agentes inmunosupresores/inmunomoduladores, no se evidenció un número significativo de hospitalizaciones o muertes asociadas a la COVID-19 ${ }^{(22)}$.

Por otra parte, en un reporte de caso de un paciente de 71 años, con psoriasis en remisión, sin lesiones cutáneas, en el cuarto día de tratamiento para COVID-19 con oseltamivir e hidroxicloroquina, se observó exacerbación de la psoriasis, lo que puede deberse a que la hidroxicloroquina es un inhibidor de la transglutaminasa epidérmica y causa la acumulación de células epidérmicas, además de que promueve la producción de IL-17, lo que da como resultado el crecimiento y la diferenciación de queratinocitos. Por tanto, los autores concluyeron que el tratamiento con hidroxicloroquina iniciada como una dosis alta el primer día puede ser el principal causante de exacerbación, dado que no hay evidencia de que el oseltamivir pueda afectar la psoriasis ${ }^{(5)}$.

Finalmente, los datos observacionales sugieren un mayor riesgo de infecciones graves con algunos agentes biológicos. En contraste, existe un papel protector teórico de los inmunomoduladores para atenuar una respuesta inflamatoria sistémica grave a la infección; sin embargo, se desconoce su seguridad relativa y las implicaciones para el riesgo o el pronóstico de pacientes dermatológicos con COVID-19. Dado lo anterior, para abordar estas brechas, las comunidades dermatológicas clínicas, científicas y de salud pública internacionales se han conectado rápidamente para recopilar datos de observación sobre los resultados de COVID-19 en la psoriasis y dermatitis atópica, a escala mundial, con las iniciativas PsoPROTECT (registro de pacientes con psoriasis para resultados, terapia y epidemiología de infección por COVID-19) y SECUREAD (epidemiología de vigilancia del coronavirus bajo investigación de dermatitis atópica de exclusión), las cuales abordan una necesidad insatisfecha de delinear los determinantes de los resultados de COVID-19 en las enfermedades inflamatorias cutáneas inmunomediadas comunes, psoriasis y dermatitis atópica. Una estrecha colaboración internacional entre especialistas, científicos y pacientes es esencial para cumplir con el objetivo de las iniciativas y obtener rápidamente conjuntos de datos a gran escala con un alto valor traslacional, conocimiento que podrá guiar el tratamiento y manejo de estos pacientes, para mitigar el riesgo potencial de COVID-19 ${ }^{(23)}$.

\section{RECOMENDACIONES}

\section{GENERALES}

1. Crear un canal de comunicación fácil para proporcionar apoyo clínico y humanizado a los pacientes con psoriasis (recomendación a favor) ${ }^{(16)}$.

2. Priorizar e individualizar los protocolos de tratamiento para pacientes con psoriasis, evaluando el riesgo/beneficio según la gravedad de la enfermedad, las condiciones clínicas de los pacientes y la gravedad del COVID-19 (recomendación a favor) ${ }^{(2,3,8,10)}$.

3. Recomendar a los pacientes tratados con terapia biológica cumplir cuidadosamente con las normas de higiene, el uso de elementos de protección personal, distanciamiento social; no suspender espontáneamente la terapia en curso e informar al dermatólogo en caso de síntomas (recomendación a favor) ${ }^{(16,24)}$.

4. Se recomienda la aplicación de terapias inmunosupresoras sistémicas o biológicas en el domicilio de aquellos pacientes que sus condiciones lo permitan (recomendación a favor) ${ }^{(8,9)}$.

\section{RECOMENDACIONES DE TRATAMIENTO DE LA PSORIASIS}

1. Se recomienda continuar la terapia biológica en pacientes que no muestran síntomas o con síntomas respiratorios leves (como tos leve sin fiebre) y sin contacto cercano con pacientes confirmados con COVID-19 (recomendación a favor) ${ }^{(2,3,8,24,25)}$. 
2. En los pacientes que requieren inicio de tratamiento con agentes inmunosupresores sistémicos se recomienda la evaluación individual de caso por caso determinando el riesgo-beneficio previo a su inicio, teniendo en cuenta que puede desarrollar complicaciones por la infección por COVID-19 (recomendación a favor) ${ }^{(25)}$.

3. Los pacientes que requieren inicio o están en tratamiento con retinoides sistémicos orales deben continuar o iniciar si los beneficios superan el riesgo. Para las mujeres en edad fértil se debe obtener el consentimiento informado al inicio del tratamiento, realizar prueba de embarazo y repetirla según la práctica clínica habitual. Para los demás pacientes es posible dar prescripción para varios meses, con seguimiento y control adecuado de análisis de sangre y efectos secundarios (recomendación a favor) ${ }^{(26)}$.

4. Considerar la optimización del tratamiento inmunosupresor en pacientes con enfermedad estable, previo acuerdo con este (recomendación a favor) $(12,27)$.

5. Se recomienda no suspender la terapia inmunosupresora/inmunomoduladora en pacientes sin infección por COVID-19, ya que el riesgo de reactivación de la patología subyacente podría agregar un factor de riesgo adicional a las infecciones (recomendación a favor) $(4,6,8,10,28)$.

6. En los casos que requieran modificaciones de la terapia biológica, considerar las diferentes opciones de manejo tales como interrupción temporal, aumento en los intervalos de la dosis, transición a una alternativa biológica, reducción o interrupción de inmunosupresores concomitantes (metotrexato) y aumento en el uso de agentes tópicos, fototerapia $u$ otros medicamentos no inmunosupresores (recomendación a favor) ${ }^{(19)}$.

\section{RECOMENDACIONES EN CASO DE APARICIÓN O SOSPECHA DE COVID-19 EN PACIENTES EN TRATAMIENTO CON PSORIASIS}

1. En pacientes que experimentan síntomas respiratorios moderados o graves (fiebre, tos y/o dificultad para respirar) sin contacto cercano con pacientes confirmados con COVID-19 se recomienda la interrupción preventiva transitoria de la terapia biológica hasta la remisión completa de los síntomas respiratorios y al menos 72 horas sin fiebre (recomendación a favor) ${ }^{(2)}$.

2. En pacientes con síntomas respiratorios leves $\mathrm{y}$ antecedentes de contacto con pacientes con COVID-19 se recomienda posponer o interrumpir el tratamiento preventivo de la terapia biológica hasta confirmar la negatividad por laboratorio para COVID-19 (recomendación a favor) ${ }^{(2,3,8,9,24,}$ 25).

3. En pacientes con síntomas respiratorios moderados a graves y contacto cercano o antecedentes de contacto con pacientes con COVID-19 o con criterios clínicos/radiológicos consistentes con esta enfermedad se recomienda la interrupción de la terapia biológica y el ingreso inmediato al hospital (recomendación a favor) ${ }^{(2)}$.

4. En pacientes con síntomas de COVID-19 y especialmente con exposición conocida o potencial, que son tratados con inmunosupresores clásicos (corticoesteroides, ciclosporina, entre otros) y antimetabolitos (azatioprina, metotrexato, ente otros), se recomienda detener el tratamiento (recomendación a favor) ${ }^{(12)}$.

5. Se recomienda considerar la suspensión de la terapia biológica en pacientes de edad avanzada

\section{Puntos clave}

- $\quad$ En pacientes sin infección por COVID-19, no se recomienda suspender la terapia inmunosupresora/inmunomoduladora, ya que la posibilidad de reactivación de la patología subyacente, podría agregar un factor de riesgo adicional a la infección.

- Los pacientes con psoriasis y COVID-19, requieren una evaluación individual de la relación riesgo-beneficio para cualquier intervención terapéutica inmunosupresora.

- Para comprender el papel de la respuesta inmunitaria en el resultado de la COVID-19 en pacientes con psoriasis, se requiere de estudios con mayor nivel de evidencia. 
con morbilidades coexistentes, como hipertensión, diabetes y obesidad, cuando estos desarrollen síntomas similares o específicos de COVID-19 y si están expuestos a contacto con personas infectadas por el virus (recomendación a favor) ${ }^{(6)}$.

6. Se recomienda no tratar con hidroxicloroquina a los pacientes con psoriasis y diagnóstico de COVID-19, por posible exacerbación de la enfermedad de base (recomendación a favor) ${ }^{(5)}$.

7. Reiniciar la terapia inmunosupresora sistémica después de asegurarse de que los pacientes en quienes se suspendió por diagnóstico positivo para COVID-19 se hayan recuperado del proceso infeccioso (recomendación a favor) ${ }^{(25)}$.

\section{CONCLUSIONES}

Aunque la evidencia todavía es insuficiente para determinar cómo la infección por COVID-19 afectará el curso clínico de las afecciones inflamatorias crónicas como la psoriasis, y para estimar los riesgos potenciales asociados con el tratamiento sistémico, en estos pacientes se debe evaluar cuidadosamente caso por caso la relación riesgo-beneficio de cualquier intervención terapéutica inmunosupresora. Es posible considerar la suspensión de los medicamentos inmunosupresores en aquellos diagnosticados con COVID-19 ${ }^{(29)}$.

Se requiere de estudios con mayor nivel de evidencia para comprender el papel de la respuesta inmunitaria en el resultado de la COVID-19 en pacientes con psoriasis.

\section{REFERENCIAS}

1. Mugheddu C, Pizzatti L, Sanna S, Atzori L, Rongioletti F. COVID-19 pulmonary infection in erythrodermic psoriatic patient with oligodendroglioma: safety and compatibility of apremilast with critical intensive care management. J Eur Acad Dermatology Venereol. 2020;34(8):e376-e378. doi: 10.1111/jdv.16625

2. Di Lernia V. Reply: "Biologics for psoriasis during COVID-19 outbreak". J Am Acad Dermatol. 2020;82(6):e217-8. doi: 10.1016/j.jaad.2020.04.004

3. Academia Española de Dermatología y Venereología (AEDV). Comunicado del Grupo de Psoriasis de la AEDV sobre la pandemia por COVID-19 [Internet]. 2020. Disponible en: https://aedv.es/comunicado-del-grupo-de- psoriasis-de-la-aedv-sobre-la-pandemia-porcovid-19/

4. Lebwohl M, Rivera-Oyola R, Murrell DF. Should biologics for psoriasis be interrupted in the era of COVID-19? J Am Acad Dermatol. 2020;82(5):1217-8. doi: 10.1016/j.jaad.2020.03.031

5. Kutlu Ö, Metin A. A case of exacerbation of psoriasis after oseltamivir and hydroxychloroquine in a patient with COVID-19: Will cases of psoriasis increase after COVID-19 pandemic? Dermatol Ther. 2020;e13383. doi: 10.1111/dth.13383

6. AmerioP, PrignanoF, GiulianiF, GualdiG.COVID19 and psoriasis: Should we fear for patients treated with biologics? Dermatol Ther. 2020;e13434. doi: 10.1111/dth.13434

7. Tao J, Song Z, Yang L, Huang C, Feng A, Man X. Emergency management for preventing and controlling nosocomial infection of the 2019 novel coronavirus: implications for the dermatology department. Br J Dermatol. 2020;182(6):1477-8. doi: 10.1111/bjd.19011

8. Grupo Colombiano de Psoriasis. COVID-19: Posición frente a terapias sistémicas en pacientes con psoriasis [Internet]. Disponible en: https://bit. ly/32lFksD

9. National Institute for Health and Care Excellence. COVID-19 rapid guideline: dermatological conditions treated with drugs affecting the immune response. NICE. 2020. Disponible en: https://www.nice.org.uk/guidance/ng169

10. 10. Abdelmaksoud A, Goldust M, Vestita M. Comment on "COVID-19 and psoriasis: Is it time to limit treatment with immunosuppressants? A call for action”. Dermatol Ther. 2020;e1336o. doi: 10.1111/dth.13360

11. Murrell DF, Rivera-Oyola R, Lebwohl M. Reply to: "Biologics for psoriasis during COVID-19 outbreak”. J Am Acad Dermatol. 2020;82(6):e219. doi: 10.1016/j.jaad.2020.04.014

12. Price KN, Frew JW, Hsiao JL, Shi VY. COVID-19 and immunomodulator/immunosuppressant use in dermatology. J Am Acad Dermatol. 2020;82(5):e173-5. doi: 10.1016/j.jaad.2020.03.046

13. 13. Conforti C, Giuffrida R, Dianzani C, Di Meo N, Zalaudek I. COVID-19 and psoriasis: is it time to limit treatment with immunosuppressants? A call for action. Dermatol Ther. 2020;e13298. doi: 10.1111/dth.13298 
14. Bardazzi F, Loi C, Sacchelli L, Di Altobrando A. Biologic therapy for psoriasis during the covid-19 outbreak is not a choice. J Dermatolog Treat. 2020;31(4):320-1. doi: 10.1080/09546634.2020.1749545

15. Coletto L, Favalli E, Caporali R. Psoriasis and Psoriatic Arthritis: how to manage Immunosuppressants in COVID-19 days. Dermatol Ther. 2020;e13415. doi: 10.1111/dth.13415

16. Carugno A, Gambini DM, Raponi F, Vezzoli P, Locatelli AGC, Di Mercurio M, et al. COVID-19 and biologics for psoriasis: a high-epidemic area experience - Bergamo, Lombardy, Italy. J Am Acad Dermatol. 2020;83(1):292-4. doi: 10.1016/j.jaad.2020.04.165

17. Conti A, Lasagni C, Bigi L, Pellacani G. Evolution of COVID-19 infection in 4 psoriatic patients treated with biological drugs. J Eur Acad Dermatology Venereol. 2020;34(8):e360-1. doi: 10.1111/jdv.16587

18. Torres T, Puig L. Managing Cutaneous ImmuneMediated Diseases During the COVID-19 Pandemic. Am J Clin Dermatol. 2020;21(3):307-311. doi: 10.1007/s40257-020-00514-2

19. Brownstone ND, Thibodeaux QG, Reddy VD, Myers BA, Chan SY, Bhutani T, et al. Novel Coronavirus Disease (COVID-19) and Biologic Therapy in Psoriasis: Infection Risk and $\mathrm{Pa}$ tient Counseling in Uncertain Times. Dermatol Ther (Heidelb). 2020;10(3):1-11. doi: 10.1007/s13555-020-00377-9

20. Messina F, Piaserico S. SARS-CoV-2 infection in a psoriatic patient treated with IL-23 inhibitor. J Eur AcadDermatologyVenereol.2020;34(6):e254-5.doi: 10.1111/jdv.16468

21. 21. Balestri R, Rech G, Girardelli CR. SARS-CoV-2 infection in a psoriatic patient treated with IL-17 inhibitor. J Eur Acad Dermatology Venereol. 2020;34(8):e357-8. doi: 10.1111/jdv.16571

22. Gisondi P, Facheris P, Dapavo P, Piaserico S, Conti A, Naldi L, et al. The impact of COVID-19 pandemic on patients with chronic plaque psoriasis being treated with biologic therapy: The Northern Italy experience. Br J Dermatol. 2020;183(2):373-4. doi: 10.1111/bjd.19158

23. Mahil SK, Yiu ZZN, Mason KJ, Dand N, Coker $\mathrm{B}$, Wall D, et al. Global reporting of cases of COVID-19 in psoriasis and atopic dermatitis: an opportunity to inform care during a pan- demic. Br J Dermatol. 2020;183(2):404-6. doi: 10.1111/bjd.19161

24. Academia Española de Dermatología y Venereología. Comunicado GEDEAS: Recomendaciones para pacientes con enfermedades autoinmunes durante la pandemia COVID-19. AEDV. 2020. Disponible en: https://bit.ly/32kzM1j

25. American Academy of Dermatology Association. Guidance on the use of immunosuppressive agents. AADA. 2020. Disponible en: https://bit.ly/3htDRGR

26. Academia Española de Dermatología y Venereología. Comunicado GEDP: Manejo de pacientes que precisan tratamiento con retinoides orales durante la pandemia de COVID-19. AEDV. 2020. Disponible en: https://bit.ly/31qQ89n

27. Shi L, Lian N, Liu L, Chen M. Tapering and Discontinuation of Systemic Medications in Psoriasis Patients with Low Disease Activity. Dermatol Ther. 2020;e13599. doi: 10.1111/dth.13599

28. Atzori L, Mugheddu C, Addis G, Sanna S, Satta $\mathrm{R}$, Ferreli $\mathrm{C}$, et al. Psoriasis health care in the time of the coronavirus pandemic: insights from dedicated centers in Sardinia (Italy). J Eur Acad Dermatology Venereol. 2020;34(6):e247-8. doi: 10.1111/jdv.16473

29. Gisondi P, Piaserico S, Conti A, Naldi L. Dermatologists and SARS-CoV-2: The impact of the pandemic on daily practice. J Eur Acad Dermatology Venereol. 2020;34(6):1196-201. doi: 10.1111/jdv.16515 


\section{Recomendaciones generales}

1. Crear un canal de comunicación fácil para proporcionar apoyo clínico y humanizado a los pacientes con psoriasis.

2. Priorizar e individualizar los protocolos de tratamiento para pacientes con psoriasis evaluando el riesgobeneficio según la gravedad de la enfermedad, las condiciones clínicas de los pacientes y la gravedad de la COVID-

19.

3. Recomendar a los pacientes tratados con terapia biológica cumplir cuidadosamente con las normas de higiene, el uso de elementos de protección personal, distanciamiento social; no suspender espontáneamente la terapia en curso e informar al dermatólogo en caso de síntomas.

4. Se recomienda la aplicación de terapias inmunosupresoras sistémicas o biológicas en el domicilio de aquellos pacientes que sus condiciones lo permitan.

Recomendaciones de tratamiento de la psoriasis

1. Se recomienda continuar la terapia biológica en pacientes que no muestran síntomas o con síntomas respiratorios leves (como tos leve sin fiebre) y sin contacto cercano con pacientes confirmados con COVID-19.

2. En los pacientes que requieren inicio de tratamiento con agentes inmunosupresores sistémicos se recomienda la evaluación individual de caso por caso determinando el riesgo-beneficio previo a su inicio, teniendo en cuenta que pueden desarrollar complicaciones por la infección por COVID-19.

3. Los pacientes que requieren inicio o están en tratamiento con retinoides sistémicos orales deben continuar o iniciar si los beneficios superan el riesgo. Para las mujeres en edad fértil se debe obtener el consentimiento informado al inicio del tratamiento, realizar prueba de embarazo y repetirla según la práctica clínica habitual. Para los demás pacientes es posible dar recetas para varios meses, con seguimiento y control adecuado de análisis de sangre y efectos secundarios.

4. Considerar la optimización del tratamiento inmunosupresor en pacientes con enfermedad estable, previo acuerdo con estos.

5. Se recomienda no suspender la terapia inmunosupresora/inmunomoduladora en pacientes sin infección por COVID-19, ya que el riesgo de reactivación de la patología subyacente podría agregar un factor de riesgo adicional a las infecciones.

6. En los casos que requieren modificaciones de la terapia biológica, considerar las diferentes opciones de manejo tales como interrupción temporal, aumento en los intervalos de la dosis, transición a una alternativa biológica, reducción o interrupción de inmunosupresores concomitantes (metotrexato) y aumento en el uso de agentes tópicos, fototerapia u otros medicamentos no inmunosupresores.

Recomendaciones en caso de aparición o sospecha de COVID-19 en paciente en tratamiento con psoriasis

1. En pacientes que experimentan síntomas respiratorios moderados o grave (fiebre, tos y/o dificultad para respirar) sin contacto cercano con pacientes confirmados con COVID-19 se recomienda la interrupción preventiva transitoria de la terapia biológica hasta la remisión completa de los síntomas respiratorios y al menos 72 horas sin fiebre.

2. En pacientes con síntomas respiratorios leves y antecedentes de contacto con pacientes con COVID-19 se recomienda posponer o interrumpir el tratamiento preventivo de la terapia biológica, hasta confirmar la negatividad por laboratorio para COVID-19.

3. En pacientes con síntomas respiratorios moderados a graves y contacto cercano o antecedentes de contacto con pacientes con COVID-19 o con criterios clínicos/radiológicos consistentes con esta enfermedad se recomienda la interrupción de la terapia biológica y el ingreso inmediato al hospital.

4. En pacientes con síntomas de COVID-19 y especialmente con exposición conocida o potencial, que son tratados con inmunosupresores clásicos (corticoesteroides, ciclosporina, entre otros) y antimetabolitos (azatioprina, metotrexato, entre otros), se recomienda detener el tratamiento.

5. Se recomienda considerar la suspensión de la terapia biológica en pacientes de edad avanzada con morbilidades coexistentes, como hipertensión, diabetes y obesidad, cuando estos desarrollen síntomas similares o específicos de COVID-19 y si están expuestos a contacto con personas infectadas por el virus.

6. Se recomienda no tratar con hidroxicloroquina a los pacientes con psoriasis y diagnóstico de COVID-19, por posible exacerbación de la enfermedad de base.

7. Reiniciar la terapia inmunosupresora sistémica después de asegurarse de que los pacientes en quienes se suspendió por diagnóstico positivo para COVID-19 se hayan recuperado del proceso infeccioso. 